\title{
Effect of brain radiotherapy strategies on prognosis of patients with EGFR-mutant lung adenocarcinoma with brain metastasis
}

\author{
Guangchuan Deng ${ }^{1,2}$, Yingyun Zhang ${ }^{1,2}$, Jiaojiao Ke ${ }^{3}$, Qi Wang ${ }^{1,2}$, Hongyue Qin ${ }^{1,2}$, Jianbin $\mathrm{Li}^{1,2^{*}}$ and \\ Zhenxiang $\mathrm{Li}^{1 *}$ (D)
}

\begin{abstract}
Purpose: Epidermal growth factor receptor (EGFR)-mutant lung cancers have a high risk of developing brain metastases (BM). Whole brain radiotherapy (WBRT), local radiotherapy, and WBRT + Boost are frequently used for treatment of BM. This retrospective study aimed to evaluate the difference in efficacy of these radiotherapy modes in patients with EGFR-mutant lung adenocarcinoma with BMs. Further, we determined the optimal radiotherapy regimen for patients based on Lung-molGPA.
\end{abstract}

Methods and materials: We retrospectively enrolled 232 patients with EGFR-mutant lung adenocarcinoma with BMs. Patients were divided into three groups based on the different modes of brain radiotherapy: WBRT group, local radiotherapy group, and WBRT + Boost group. Graded prognostic assessment for lung cancer using molecular markers (Lung molGPA), overall survival (OS), and intracranial progression-free survival (iPFS) were calculated. Kaplan-Meier was used to compare iPFS and OS in different groups.

Results: The median OS for the WBRT $(n=84)$, local radiotherapy $(n=65)$, and WBRT + Boost $(n=83)$ cohorts was $32.8,59.1$, and 41.7 months, respectively $(P=0.0002)$. After stratification according to the Lung-molGPA score, the median OS for the WBRT $(n=56)$, local radiotherapy $(n=19)$, and WBRT + Boost $(n=28)$ cohorts was $32.5,30.9$, and 30.8 months, respectively, in subgroup with score $1-2(P=0.5097)$. In subgroup with score $2.5-4$, the median OS for the WBRT $(n=26)$, local radiotherapy $(n=45)$, and WBRT + Boost $(n=54)$ cohorts was $32,68.4$, and 51 months, respectively $(P=0.0041)$.

Conclusion: The present study showed that in patients with EGFR-mutant lung adenocarcinoma with BM, local radiotherapy and WBRT + Boost perform similarly well both in the subgroups with low and high scores of Lung-molGPA. Considering the side effect caused by whole brain radiotherapy, we recommended local radiotherapy as optimal brain radiation mode for those subtype lung cancer patients.

Keywords: EGFR-mutant, Lung adenocarcinoma, Brain metastasis, Lung-molGPA, Brain radiotherapy

*Correspondence: lijianbin@msn.com; lizx0108@163.com

1 Department of Radiation Oncology, Shandong Cancer Hospital and Institute, Shandong First Medical University and Shandong Academy of Medical Sciences, Jinan 250117, China

Full list of author information is available at the end of the article

\section{Introduction}

Advanced lung adenocarcinoma is increasingly being treated with individualized molecular targeted therapy based on gene aberrations, and the mutant epidermal growth factor receptor (EGFR) is the most common therapeutic target [1-3]. For EGFR-mutant non-small cell lung cancers (NSCLC), brain metastasis (BM) is a give appropriate credit to the original author(s) and the source, provide a link to the Creative Commons licence, and indicate if changes were made. The images or other third party material in this article are included in the article's Creative Commons licence, unless indicated otherwise in a credit line to the material. If material is not included in the article's Creative Commons licence and your intended use is not permitted by statutory regulation or exceeds the permitted use, you will need to obtain permission directly from the copyright holder. To view a copy of this licence, visit http://creativecommons.org/licenses/by/4.0/. The Creative Commons Public Domain Dedication waiver (http://creativecommons.org/publicdomain/zero/1.0/) applies to the data made available in this article, unless otherwise stated in a credit line to the data. 
severe complication in approximately $60 \%$ of patients during the course of the disease [4-6]. A previous study suggests that patients with BM have relatively low quality of life and shorter survival of only 3-6 months when untreated [7]. However, when treated with targeted therapy, patients with EGFR-mutant NSCLC with BM show median overall survival (OS) of 19 to 58 months $[8,9]$. In patients with NSCLC harboring EGFR mutations, EGFR tyrosine kinase inhibitors (TKIs) exhibit some therapeutic efficacy against BM, however they show limited intracranial progression-free survival (iPFS) of 8 to 10 months $[10,11]$.

Brain radiotherapy, an effective therapeutic method, is critical in the treatment of brain metastases [12]. The most commonly administered modes of brain radiotherapy include whole brain radiotherapy (WBRT), local radiotherapy, and WBRT + Boost $[13,14]$. Of these, WBRT is usually recommended for treatment of multiple BMs. However, WBRT can destroy the blood brain barrier (BBB) that greatly reduces the absorption of chemotherapy or targeted therapy and increases the concentration of EGFR TKIs in the cerebrospinal fluid, and also controls subclinical lesions. [12, 15-17]. Additionally, WBRT can worsen cognitive function and health-related quality of life $[17,18]$. Radiotherapy targeting local metastases can reduce radiation damage to the surrounding normal brain tissue, and thus, reduces neurotoxicity. However, it can only target metastases in the radiation field, is limited to improve the control effect of multiple intracranial metastases, and therefore, is recommended only for a limited number of BMS (1 to 4) [19]. In contrast, WBRT + Boost offers advantages of both-it can control subclinical lesions with lower dose and increase the radiation dose to brain metastases as much as possible to destroy the local brain metastases [20,21]. In recent years, some studies have suggested that WBRT + Boost is superior to WBRT in lung cancer patients with craniocerebral metastasis. However, those studies involved in mixed pathological type especially small cell lung cancer and did not take gene status into account to determine the therapy choice. [14, 20, 22].

The choice of brain radiotherapy mode should be based on the number of metastatic sites and overall condition of the patient. A recent study used graded prognostic assessment for lung cancer using molecular markers (Lung-molGPA) to evaluate patients according to age, Karnofsky Performance Status (KPS), extracranial metastasis, number of brain metastasis, and gene mutation status; a score of $0-4$ indicated significant impact on OS in patients with NSCLCs with BMs, which may also influence the selection of radiotherapy mode [23, 24]. A study found that patients receiving local radiotherapy had an OS of 64 months (95\% CI: 46 to not reach) with a better prognosis (DS-GPA, 2-4), while those receiving WBRT had OS of 52 months (95\% CI 32 to 79) with the same prognosis (DS-GPA, 2-4) and those who received EGFRTKI followed by RT at intracranial progression only had OS of 32 months (95\% CI 26 to 39) [2].

Previous studies have showed that combining brain radiotherapy with EGFR TKIs can further increase local control of intracranial lesions. However, only few studies have explored the different outcomes in patients with EGFR-mutant lung cancer who received different radiotherapy modes, especially after considering the LungmolGPA. Therefore, we performed this retrospective, real-world analysis for EGFR-mutant lung adenocarcinomas with BM to confirm the optimal brain radiotherapy regimen.

\section{Methods and materials Patient cohort}

We screened $>800$ patients diagnosed with NSCLC at our hospital between March 2008 and December 2019. The inclusion criteria were as follows: (1) pathologically diagnosed with primary lung adenocarcinoma; (2) mutation in EGFR exon 18,19, or 21; (3) diagnosis of BM by enhanced computed tomography (CT) or magnetic resonance imaging (MRI); (4) detailed clinical information, including treatment options and clinicopathological features; (5) EGFR TKIs administered (e.g. gefitinib, erlotinib, or icotinib); (6) previously received brain radiotherapy, including WBRT, local radiotherapy, or WBRT + Boost; and (7) no other primary malignancies. Patients with incomplete medical records or those who failed to meet the above criteria were excluded from the study. The study was approved by the Ethics Committee of the of the Shandong Cancer Hospital, and was conducted in accordance with the Declaration of Helsinki.

The following characteristics were collected for analysis: age, sex, smoking history, EGFR mutation status, BM during initial diagnosis, and treatment. The start date of initial local therapies, start of EGFR-TKIs, intracranial progression, most recent follow-up, and death were recorded. Intracranial progression was defined as radiographic progression of preexisting BM, development of new BM, or both. OS was calculated from the date of pathological diagnosis of lung adenocarcinoma to date of death or reexamination at the last follow-up. Intracranial PFS was measured and reviewed at the last followup. The time to intracranial progression was calculated from the date of the start of brain radiotherapy to date of intracranial progression.

Lung-molGPA is a specific, graded prognostic assessment based on the patient's age, KPS, the number of extracranial and $\mathrm{BM}$, and the status of gene mutations [25]. The scoring criteria are shown in Table 1. 
Table 1 Lung-molGPA

\begin{tabular}{llll}
\hline Prognostic & $\mathbf{0}$ & $\mathbf{0 . 5}$ & $\mathbf{1 . 0}$ \\
\hline Age $(\mathrm{y})$ & $\geq 70$ & $<70$ & NA \\
KPS & $<70$ & 80 & $90-100$ \\
ECM & Present & & Absent \\
Brain metastases, No. & $>4$ & $1-4$ & NA \\
Gene status & EGFR neg/unk and & NA & EGFR pos \\
& ALK neg/unk & & Or ALK \\
& & & pos \\
\hline
\end{tabular}

GPA: graded prognostic assessment, ECM extracranial metastases, KPS Karnofsky Performance Status, NA not applicable, neg/unk negative or unknown, pos positive

\section{EGFR genotyping}

Genomic DNA was extracted from tissues obtained by fiberoptic bronchoscopy or puncture biopsy. In contrast, circulating tumor DNA (CtDNA) was isolated and purified from the blood. EGFR mutations were detected by next-generation sequencing (NGS), droplet digital polymerase chain reaction (ddPCR), or amplification refractory mutation system (ARMS)-PCR.

\section{Radiotherapy}

In patients treated with whole brain radiotherapy, the median prescribed dose was 40 Gy (range, 30-50 Gy). The median prescribed dose for local radiotherapy was 50 Gy (range, 30-62.5 Gy). Additionally, in the WBRT + Boost group, the median prescription dose of whole brain was 40 Gy (range, 22-50 Gy). The median dose of the additional radiation boost for local metastases was 15 Gy (range, 6-50 Gy).

\section{Statistical methods}

The characteristics of each group were descriptively compared, and the classified variables were tested by chisquare test. Kaplan-Meier method was used for survival analysis, and log rank test was used to test the influence of individual variables on survival. A $p$ value $<0.05$ (twosided) was considered to be statistically significant. Analyses were performed using GraphPad Prism version 8.0.1.

\section{Results}

\section{Patients characteristics}

A total of 232 patients who met the inclusion criteria were enrolled in the study. According to the brain radiotherapy scheme, the patients were divided into three groups: WBRT, local radiotherapy, and WBRT + Boost. 84,65 , and 83 patients received WBRT, local radiotherapy, and WBRT + Boost, respectively. The characteristics of the patients are detailed in Table 2. The median age at diagnosis was 54 (range, 28-81) years. A majority of the patients were women $(149,64.2 \%)$ and non-smokers $(182,78.4 \%)$. As a first-line treatment, $46.6 \%$ of the patients (108/232) received EGFR TKIs, while 53.4\% $(124 / 232)$ received platinum-based treatment. More than $50 \%$ of the patients $(62.1 \%)$ had craniocerebral metastasis at the time of initial diagnosis. Moreover, mutations in EGFR exons 19 and 21 were detected in 41.4\% (96/232) and $49.6 \%(115 / 232)$ of the patients, respectively.

\section{Survival outcomes for the entire cohort in the study}

As of March 2021, 159 deaths (68.5\%) were recorded. The median follow-up duration was 60.1 (interquartile range, 48.0 to 90.6 ) months. Median OS was 37.5 months, while median iPFS was 16.2 months (Fig. 1a, b). There was a trend for difference in OS between groups with mutations in exons 19 and 21 (40.7 months vs. 35.8 months; $\log$-rank $p=0.0882$; HR: 0.7559 ; $95 \%$ CI 0.5465 to 1.046 ; Fig. 1c). The median OS in the patients with $\mathrm{BM}$ at the initial diagnosis and those with BM during the treatment was 36.1 months and 41.6 months, respectively (log-rank $p=0.3495$; HR: 1.161 ; 95\% CI 0.8491 to 1.589 ; Fig. $1 d$ ).

The distribution predicted by Lung-molGPA in the three radiotherapy groups was unequal. According to the grading criteria, patients with scores $1-2$ were classified into group A, while those with scores $2.5-4$ were classified into group B for statistical analysis. The median survival time of patients in groups A and B was 32.3 months and 48 months, respectively (log-rank $p<0.0001$; HR: 1.925 ; $95 \%$ CI 1.390 to 2.667; Fig. 1e).

\section{Effects of different radiotherapy modes on iPFS}

Of the 232 patients who received radiotherapy, 184 had intracranial progression after radiotherapy (WBRT group, 76; local radiotherapy group, 44; and WBRT + Boost group, 64). The iPFS analysis was performed with WBRT, local radiotherapy, and WBRT + Boost groups. The median iPFS in the WBRT, local radiotherapy, and WBRT + Boost groups was 13, 16.2, and 18.7 months, respectively (Fig. 2a); and, there were significant differences in median iPFS between the WBRT and local radiotherapy groups $(p=0.0421$; HR: 1.534; $95 \%$ CI 1.027 to 2.290; Fig. 2b), and WBRT and WBRT + Boost groups (log-rank $p=0.0014$; HR: 1.765; 95\% CI 1.221 to 2.550; Fig. 2c). Therefore, the iPFS in patients receiving local radiotherapy or WBRT + Boost was better than that in patients receiving WBRT. However, there was no significant difference in iPFS between the local radiotherapy and WBRT + boost groups (logrank $p=0.5803$; HR: $0.8861 ; 95 \%$ CI 0.5706 to 1.376 ; Fig. 2d).

Further, as Lung-molGPA had a significant impact on the prognosis of patients, we performed an iPFS analysis between the three groups stratified by 
Table 2 Characteristics of 232 NSCLC patients and with chi-square test for categorical variables

\begin{tabular}{|c|c|c|c|c|}
\hline Characteristics & $\begin{array}{l}\text { WBRT } \\
(n=84)\end{array}$ & Local Radiotherapy $(n=65)$ & WBRT + Boost $(n=83)$ & $P$ value \\
\hline Age, years & $53(28-77)$ & $56(38-81)$ & $53(33-78)$ & \\
\hline$>60$ & $21(25.0)$ & $24(36.9)$ & $23(27.7)$ & 0.263 \\
\hline$\leq 60$ & $63(75.0)$ & $41(63.1)$ & $60(72.3)$ & \\
\hline \multicolumn{5}{|l|}{ Sex } \\
\hline Female & $51(60.7)$ & $43(66.2)$ & $55(66.3)$ & 0.703 \\
\hline Male & $33(39.3)$ & $22(33.8)$ & 28(33.7) & \\
\hline \multicolumn{5}{|l|}{ Smoking status } \\
\hline Never & $61(72.6)$ & 48(73.8) & 73(88.0) & 0.031 \\
\hline Former/current & $23(27.4)$ & $17(26.2)$ & 10(12.0) & \\
\hline \multicolumn{5}{|l|}{ EGFR mutation } \\
\hline Exon 18 & $1(1.2)$ & 0 & $2(2.4)$ & 0.462 \\
\hline Exon 19 & $36(42.9)$ & 24(36.9) & $36(43.4)$ & \\
\hline Exon 21 & $41(48.8)$ & $38(58.5)$ & $36(43.4)$ & \\
\hline Unclear & $6(7.1)$ & $3(4.6)$ & $9(10.8)$ & \\
\hline \multicolumn{5}{|l|}{ Systemic therapy } \\
\hline First-line EGFR TKIs & $38(45.2)$ & $30(46.2)$ & $40(48.2)$ & 0.927 \\
\hline Second-line EGFRTKIs & $46(54.8)$ & $35(53.8)$ & 43(51.8) & \\
\hline \multicolumn{5}{|l|}{ Lung-molGPA } \\
\hline $1-2$ & $56(66.6)$ & 19(29.2) & 28(33.7) & $<0.001$ \\
\hline $2.5-3$ & $24(28.6)$ & $33(50.8)$ & $41(49.4)$ & \\
\hline $3.5-4$ & $2(2.4)$ & 12(18.5) & 13(15.7) & \\
\hline Unclear & $2(2.4)$ & $1(1.5)$ & $1(1.2)$ & \\
\hline \multicolumn{5}{|l|}{ Brain metastatic time } \\
\hline Initial treatment & $47(56.0)$ & $41(63.1)$ & $56(67.5)$ & 0.303 \\
\hline In the course of treatment & $37(44.0)$ & $24(36.9)$ & $27(32.5)$ & \\
\hline
\end{tabular}

EGFR epidermal growth factor receptor, TKI tyrosine kinase inhibitors

Lung-molGPA score. For patients in the group A, the median iPFS was 13.2, 9.65, and 18.2 months (Fig. 2e). The difference in iPFS between the WBRT and local radiotherapy groups was not statistically significant (log-rank $p=0.8137$; HR: 1.071 ; $95 \%$ CI 0.5930 to 1.934 ; Fig. 2f), but that between WBRT and WBRT + Boost groups trended towards statistically significant (logrank $p=0.0529$; HR: $1.657 ; 95 \%$ CI 1.010 to 2.718 ; Fig. $2 \mathrm{~g}$ ). Moreover, there was no significant difference between the WBRT + Boost and local radiotherapy groups (log-rank $p=0.2728$; HR: $1.438 ; 95 \%$ CI 0.7026 to 2.944; Fig. 2h).

For patients in the group B, the median iPFS of the three groups was 13, 26.1, and 20.6 months (Fig. 2i). The difference between the WBRT and local radiotherapy groups was statistically significant (log-rank $p=0.0109$; HR: 2.200 ; $95 \%$ CI 1.090 to 4.442; Fig. 2j), and that between the WBRT and WBRT + Boost groups was also statistically significant (log-rank $p=0.0245$; HR: 1.863 ; $95 \%$ CI 0.9672 to 3.590; Fig. 2k). However, the difference between WBRT + Boost and local radiotherapy groups was not statistically significant $(\log -\operatorname{rank} p=0.7203$; HR: 1.106; $95 \%$ CI 0.6335 to 1.932; Fig. 2l).

\section{Effects of different radiotherapy modes on OS}

The analysis of OS in patients in the WBRT, local radiotherapy, and WBRT + Boost groups indicated median OS to be 32.8, 59.1, and 41.7 months, respectively (Fig. 3a). There was a significant difference in the median OS between the WBRT and local radiotherapy groups (log-rank $p<0.0001$; HR: 2.209 ; $95 \%$ CI 1.495 to 3.265; Fig. $3 \mathrm{~b}$ ), and WBRT and WBRT + Boost groups (log-rank $p=0.0030$; HR: 1.660 ; $95 \%$ CI 1.163 to 2.369 ; Fig. 3c). However, there was no significant difference in OS between the local radiotherapy and WBRT + Boost groups (log-rank $p=0.1685$; HR: 0.7458; 95\% CI 0.4950 to 1.124; Fig. 3d).

Furthermore, patients were divided into group A and group $\mathrm{B}$ according to the Lung-molGPA score. The median OS of the WBRT, local radiotherapy, and WBRT + Boost groups was $32.5,30.9$, and 30.8 months, respectively, for patients in the group A. Moreover, there 

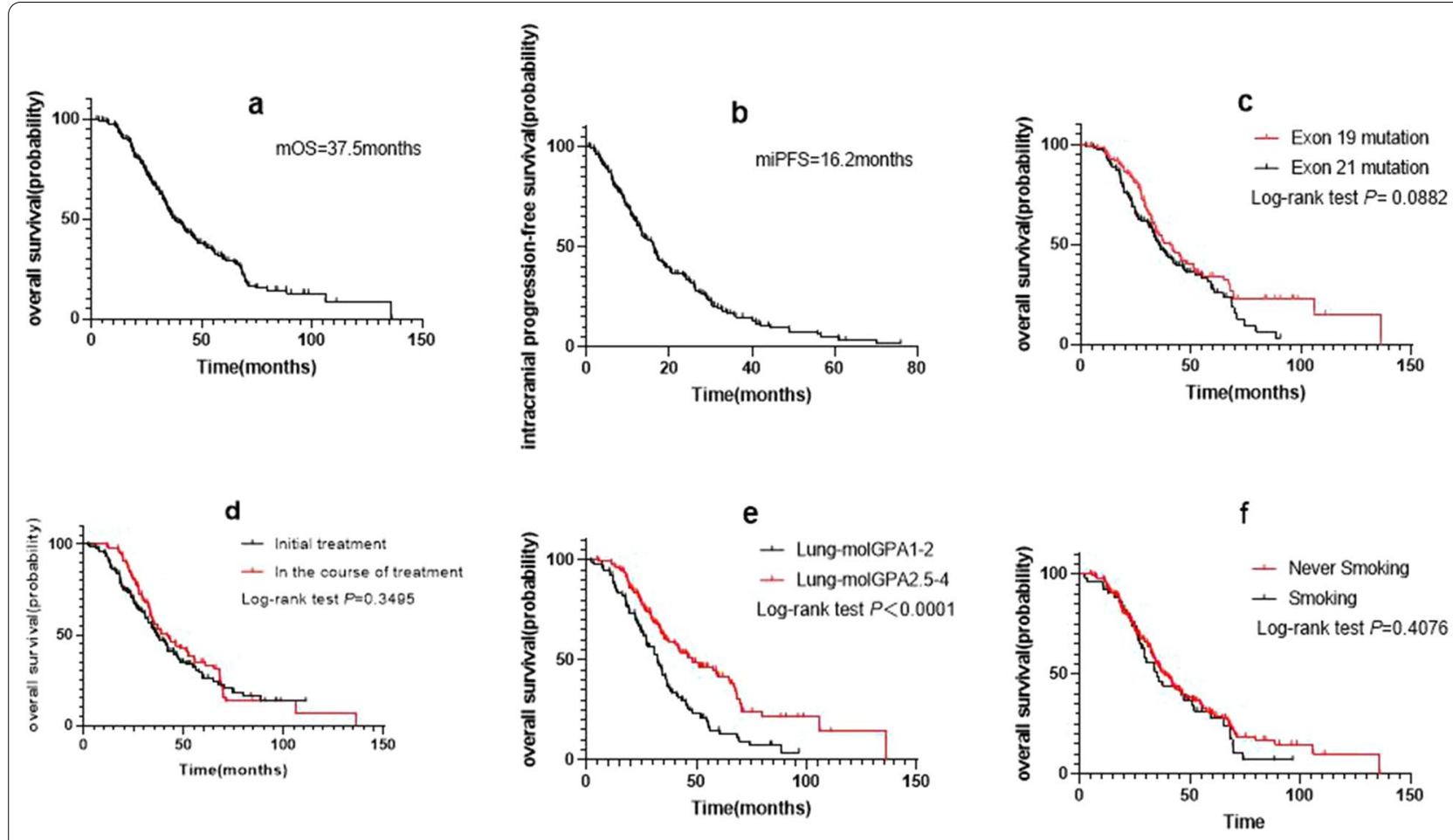

Fig. 1 a Overall survival (OS) of the entire cohort. b Intracranial progression-free survival (iPFS) of the entire cohort. OS of patients stratified according to $\mathbf{c} E G F R$ mutation status, $\mathbf{d}$ time of occurrence of brain metastasis, e Lung-molGPA, and $\mathbf{f}$ Smoking Status

was no significant difference in median OS between the three brain radiotherapy regimens $(\log$-rank $p=0.5097$, Fig. 3e). There was also no significant difference between the WBRT and the other two groups (log-rank $p=0.2116$, HR: $1.422,95 \%$ CI 0.8273 to 2.445 , Fig. 3f; and log-rank $p=0.6624$, HR: 1.115 , 95\% CI 0.6755 to 1.840 , Fig. $3 \mathrm{~g})$, and the local radiotherapy and WBRT + Boost groups (log-rank $p=0.5806$, HR: $0.8333,95 \%$ CI 0.4360 to 1.592 , Fig. 3h).

For patients in the group B, there was significant difference in OS between patients receiving different radiotherapy modes $(\log$-rank $p=0.0041$, Fig. 3i). The median OS of the patients was $32,68.4$, and 51 months in the different groups. The OS of patients receiving local radiotherapy or WBRT + Boost was better than that of patients receiving WBRT-alone. The difference was statistically significant between the WBRT and local radiotherapy groups (log-rank $p=0.0011, \mathrm{HR}: 2.698,95 \%$ CI 1.319 to 5.520, Fig. 3j), and the WBRT and WBRT+Boost groups (log-rank $p=0.0210$, HR: $1.878,95 \%$ CI: 0.9917 to 3.557 , Fig. 3k). However, there was no significant difference between the WBRT + Boost and local radiotherapy groups (log-rank $p=0.1968$, HR: $0.6976,95 \%$ CI 0.4091 to 1.190 , Fig. 31).

We further performed univariate and multivariate analysis as shown in Table 3. The univariate analysis results show that the effect of gender, age, smoking status, brain metastatic time on prognosis was not statistically significant. Multivariate analysis showed that only brain radiotherapy mode and Lung-molGPA were independent predictive factors $(p=0.004)$.

\section{Discussion}

The recent evolution of targeted therapies has helped prolong the OS of patients with EGFR-mutant advanced lung adenocarcinoma [26-28]. As per a previous study, the median OS was extended to 31.8 months after administration of the first generation EGFR TKIs, while the advent of the third generation EGFR TKIs extended the survival to 38.6 months. [29] However, this enhanced OS increased the probability of developing BM. Moreover, BM frequently occurred in EGFR-mutant NSCLC, with approximately $8-49 \%$ that occurred at the initial diagnosis and approximately $24 \%$ during the treatment course $[7,30]$. In the present study, $38.2 \%$ of the patients developed BM during the treatment, emphasizing the importance of local treatment, such as brain radiotherapy, in patients who develop BM after systemic treatment [17]. To the best of our knowledge, the present study is the first to compare the effects of three radiotherapy modes for $\mathrm{BM}$ on the prognosis of patients with EGFR-mutant NSCLC. 

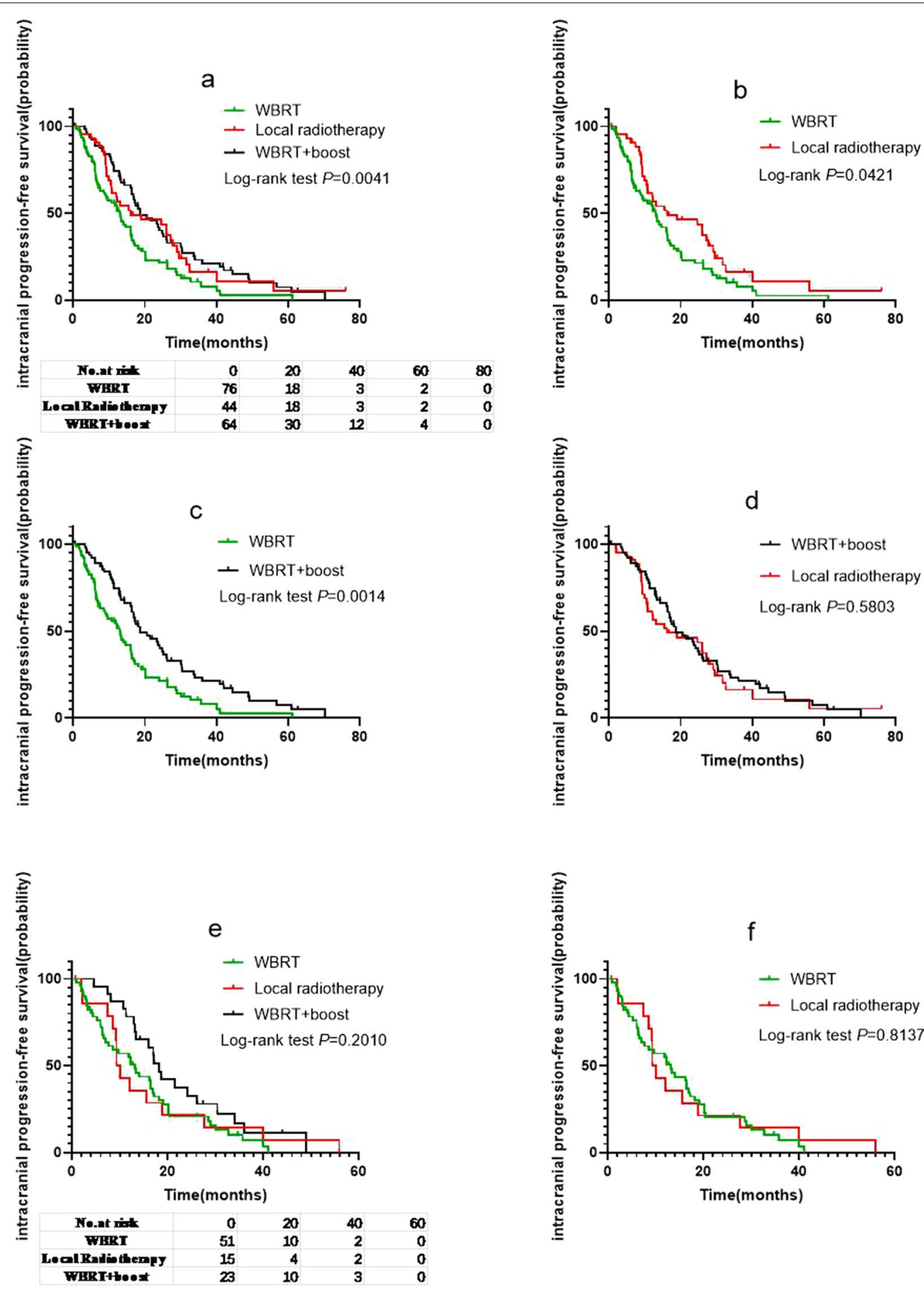

Fig. 2 a Intracranial progression-free survival (iPFS) of patients according to different the brain radiotherapy strategies. b Comparison of iPFS between the whole brain radiotherapy (WBRT) and local radiotherapy groups. c Comparison of iPFS between the WBRT and WBRT + Boost groups. $\mathbf{d}$ Comparison of iPFS between the local radiotherapy and WBRT + Boost groups. e iPFS of patients in group A stratified according to different brain radiotherapy strategies and $\mathbf{f}-\mathbf{h}$ the differences between them. i iPFS of patients in group B stratified according to different brain radiotherapy strategies and $\mathbf{j}-\mathbf{I}$ the differences between them. The results showed that in the low Lung-molGPA groups, only WBRT group and WBRT + Boost group trended towards statistically significant, while in the high Lung-molGPA groups, the difference between WBRT group and the other two groups was statistically significant 

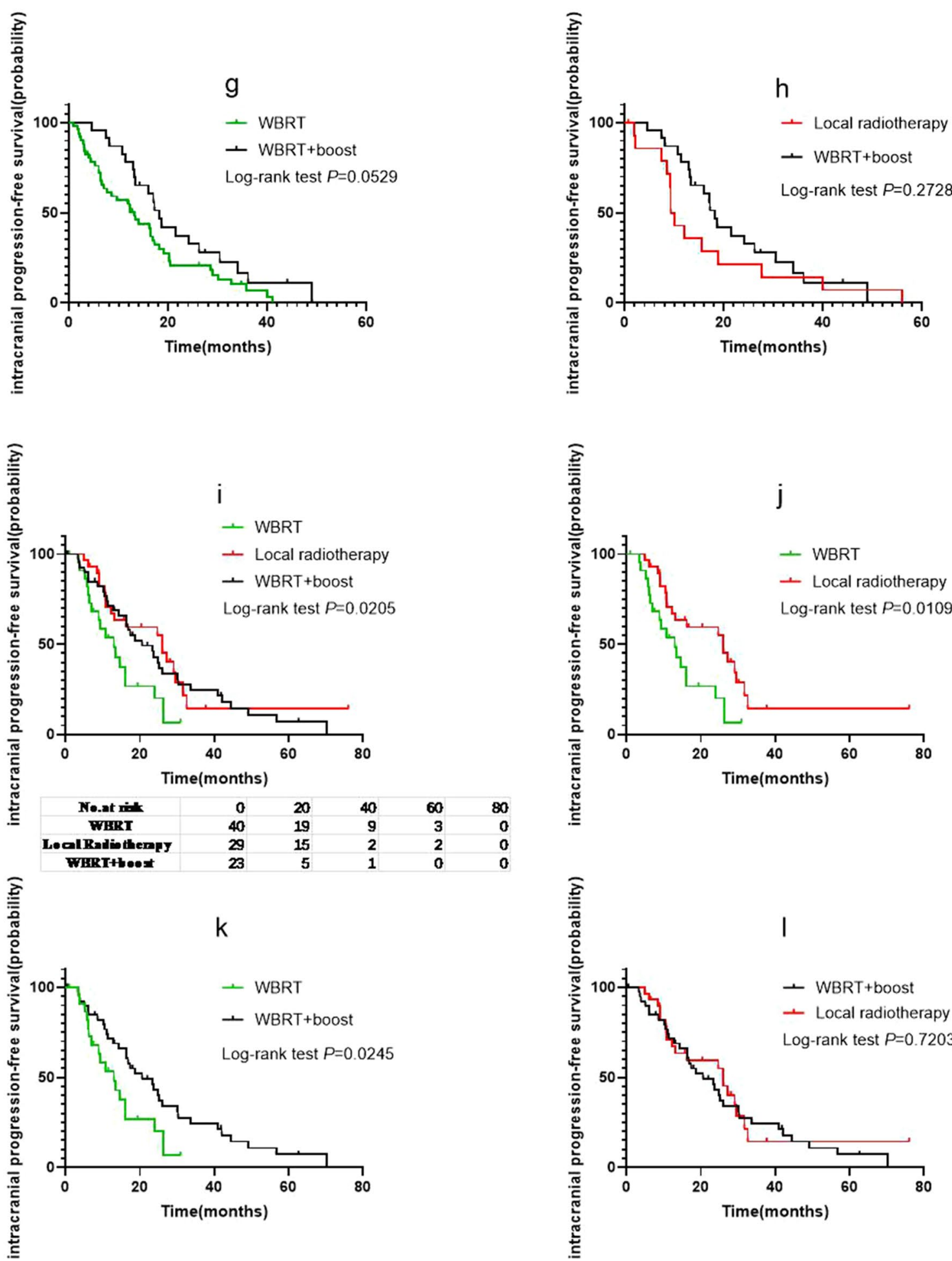

Fig. 2 continued

The number of craniocerebral metastases is closely related to the prognosis of patients with NSCLC, which plays an important role in the formulation of brain radiotherapy strategy. In clinical practice, the formulation of radiotherapy regimens is primarily based on the number of BM. For example, in patients with $>3$ 


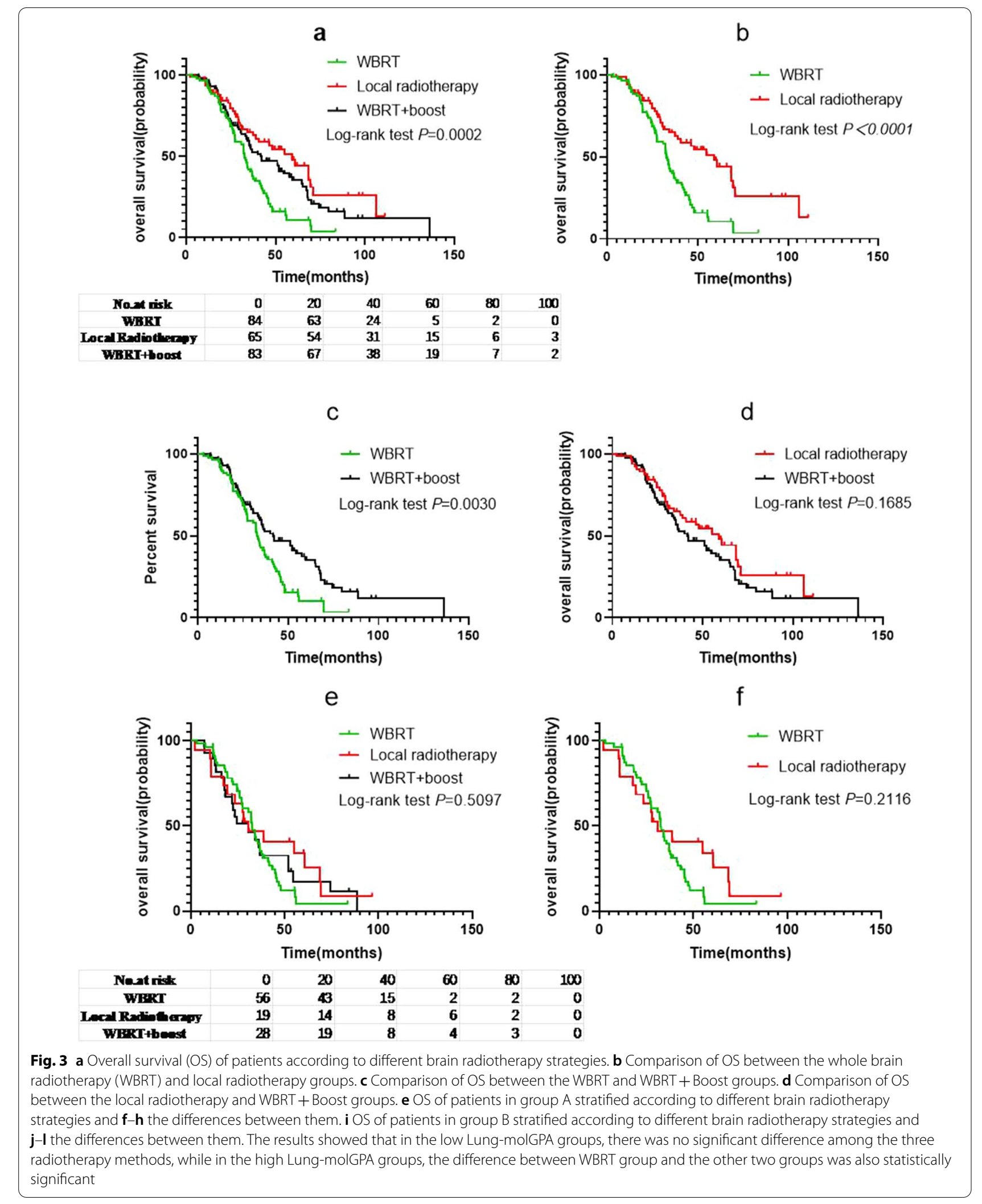




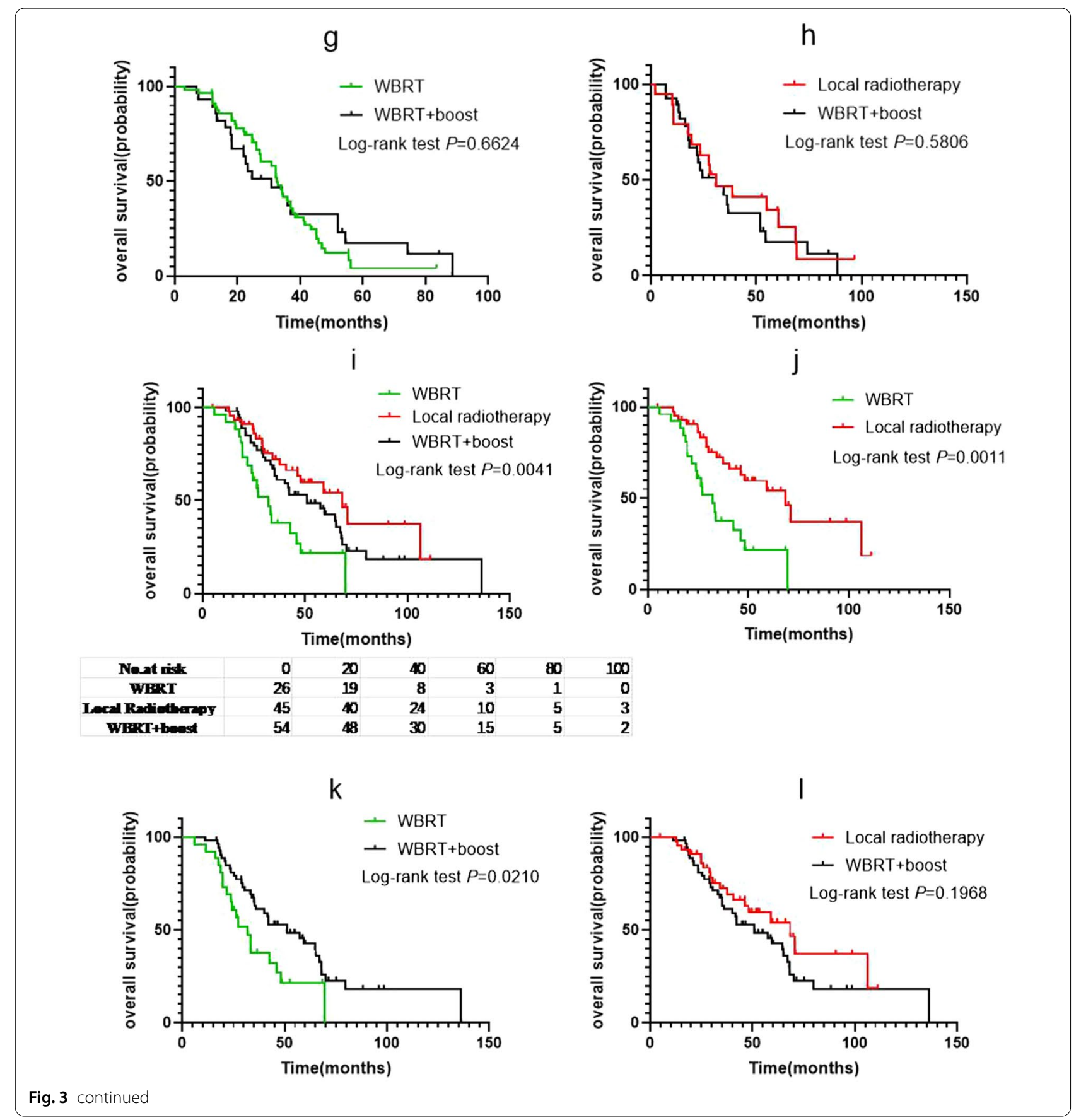

metastatic foci, WBRT was the main treatment; but, in patients with $\leq 3$ metastatic foci, local radiotherapy was selected [14]. In addition to the number of BM, recent studies have found that Lung-molGPA offers a better predictive ability and may affect decisions on the brain radiotherapy strategy [27]. This suggests that Lung-molGPA can comprehensively and accurately reflect the prognosis in patients with NSCLC with BM. In the present study, we divided the patients into two groups according to the Lung-molGPA score. Survival analysis showed that the median OS in patients in the high score group was significantly longer than that in patients in low score group (log-rank $p<0.0001)$, which was consistent with the results reported previously [27].

The proportion of patients with low scores in the WBRT group was higher (66.6\%), while that of 
Table 3 Univariable and Multivariable analyses of covariable associated with OS

\begin{tabular}{|c|c|c|c|c|c|c|}
\hline \multirow[b]{2}{*}{ Variable } & \multicolumn{3}{|c|}{ Univariable analysis } & \multicolumn{3}{|c|}{ Multivariable analysis } \\
\hline & HR & $95 \% \mathrm{Cl}$ & $\mathbf{P}$ & HR & $95 \% \mathrm{Cl}$ & $\mathbf{P}$ \\
\hline \multicolumn{7}{|l|}{ Age (y) } \\
\hline$<60$ vs $\leq 60$ & 0.959 & 0.684 to 1.344 & 0.807 & & & \\
\hline \multicolumn{7}{|l|}{ Sex } \\
\hline Female vs male & 1.014 & 0.733 to 1.402 & 0.935 & & & \\
\hline \multicolumn{7}{|l|}{ Smoking status } \\
\hline Never vs current/former & 1.136 & 0.786 to 1.643 & 0.497 & & & \\
\hline \multicolumn{7}{|l|}{ EGFR mutation } \\
\hline Exon 19 & 0.721 & 0.520 to 1.000 & 0.050 & 0.800 & 0.495 to 1.292 & 0.362 \\
\hline Exon 21 & 1.252 & 0.914 to 1.715 & 0.161 & 1.104 & 0.693 to 1.104 & 0.678 \\
\hline \multicolumn{7}{|l|}{ First-line TKI therapy } \\
\hline Yes v no & 1.480 & 1.077 to 2.032 & 0.016 & 1.358 & 0.985 to 1.872 & 0.061 \\
\hline \multicolumn{7}{|l|}{ Lung-molGPA } \\
\hline $1-2$ v $2.5-4$ & 0.513 & 0.374 to 0.703 & $<0.001$ & 0.606 & 0.433 to 0.849 & 0.004 \\
\hline \multicolumn{7}{|l|}{ Radiotherapy strategies } \\
\hline WBRT & 1.835 & 1.326 to 2.539 & $<0.001$ & 1.529 & 1.084 to 2.157 & 0.016 \\
\hline Local radiotherapy & 0.456 & 0.299 to 0.694 & $<0.001$ & 0.530 & 0.340 to 0.827 & 0.005 \\
\hline WBRT + Boost & 0.623 & 0.434 to 0.894 & 0.010 & 0.744 & 0.509 to 1.087 & 0.126 \\
\hline \multicolumn{7}{|l|}{ Brain metastatic time } \\
\hline $\begin{array}{l}\text { Initial treatment vs In the course } \\
\text { of treatment }\end{array}$ & 1.083 & 0.923 to 1.271 & 0.329 & & & \\
\hline
\end{tabular}

patients with high scores in the local radiotherapy and WBRT + boost groups was $69.3 \%$ and $65.5 \%$, respectively, indicating that the difference in the Lung-molGPA constitution ratio existed in different radiotherapy groups. To better clarify the effects of different modes of radiotherapy on the prognosis of patients, we divided the patients into two groups according to the Lung-molGPA score as the high score and low score groups. For patients with low score, there is no statistically difference of iPFS among WBRT + boost group, local radiotherapy group and WBRT group. However, the enhanced iPFS failed to translate to improved OS. Therefore, in patients with worse clinical conditions, the synchronous or sequential dose addition based on WBRT has little effect on the increase in OS. In contrast, it may indicate greater side effects that may have an adverse impact on the quality of life [31]. Therefore, it may be more reasonable for such patients to receive radiotherapy with less side effects only for treatment of metastatic lesions. A recent study suggested that the benefit of treatment with local radiotherapy is independent of the number of BM [32] Another study has shown that local radiotherapy can also achieve good local control and prognosis in patients with 5-10 BM [33]. Additionally, we emphasize the importance of systemic treatment in patients in whom the third generation of EGFR TKIs, such as osimertinib, have been widely used with a good effect on both intracranial and extracranial lesions [34, 35].

In patients with high Lung-molGPA score and better clinical condition, survival analysis showed that median iPFS in the local radiotherapy and WBRT + boost groups was longer than that in WBRT group. Moreover, there was no significant difference in iPFS between the local radiotherapy and WBRT + boost groups. The results of the OS were similar to that of iPFS. The OS in the WBRT + boost and local radiotherapy groups was better than that in WBRT group, but there were no significant differences between the WBRT + boost and local radiotherapy groups. These results suggest that the OS of patients with good prognosis is longer, and increasing the local control can further prolong the OS in patients. However, OS in the WBRT + boost high dose group with craniocerebral metastasis was not superior to that in local radiotherapy group, indicating that in patients with good prognosis, the formulation of radiotherapy strategy should consider both local control and side effects of brain radiotherapy. The WBRT + boost intervention may increase toxicity and side effects after brain radiotherapy that may adversely affect the prognosis in patients [31]. Patients with higher scores have a good overall prognosis, and EGFR TKIs are effective against BM [36]. Therefore, results of the present study suggest that the range of radiation target should be reduced in patients with higher 
Lung-molGPA scores and local radiotherapy should be administered on priority to avoid neurotoxicity of WBRT $[18,19]$.

The study has some limitations. First, it was a singlecenter, retrospective study with limited sample size, and thus, had selection bias. Second, there were differences in the type, timing, combination, and sequence of chemotherapy and targeted therapy administered in patients in the study; therefore, it was impossible to evaluate the effects of the drugs on patients. Third, the uneven dose of radiotherapy in the same group may have an impact on the prognosis of patients to some extent. Therefore, considering these limitations, we warrant a large sample size, multi-institutional, prospective study to confirm the findings of the present study.

\section{Conclusion}

The present study showed that in patients with EGFRmutant lung adenocarcinoma with BM, local radiotherapy and WBRT + Boost perform similarly well both in the subgroups with low and high scores of LungmolGPA. Considering the side effect caused by whole brain radiotherapy, we recommended local radiotherapy as optimal brain radiation mode for those subtype lung cancer patients. Prospective study will be performed to verify the findings in the study.

\footnotetext{
Authors' contributions

Conceptualization, GD and ZL; methodology, GD; software, GD; validation, $\mathrm{GD}$ and $Z \mathrm{~L}$; formal analysis, GD and ZL; resources, GD; data curation, GD, YZ, JK, QW and HQ; writing — original draft preparation, GD; writing - review and editing, GD and ZL; supervision, JL and ZL. All authors have read and approved the final manuscript.
}

\section{Funding}

Taishan Scholars Program of Shandong Province (NO.ts 20190982).

\section{Availability of data and materials}

Data are available on request to the authors.

\section{Declarations}

Ethics approval and consent to participate Not applicable.

\section{Consent for publication}

All authors are consent for publication.

\section{Competing interests}

All authors have no conflict of interest with any company or organization.

\section{Author details}

${ }^{1}$ Department of Radiation Oncology, Shandong Cancer Hospital and Institute, Shandong First Medical University and Shandong Academy of Medical Sciences, Jinan 250117, China. ${ }^{2}$ Shandong First Medical University and Shandong Academy of Medical Sciences, Jinan, People's Republic of China. ${ }^{3}$ Weihai Central Hospital, Weihai, People's Republic of China.
Received: 15 August 2021 Accepted: 22 November 2021

Published online: 30 November 2021

\section{References}

1. Lee JH, Chen HY, Hsu FM, et al. Cranial irradiation for patients with epidermal growth factor receptor (EGFR) mutant lung cancer who have brain metastases in the era of a new generation of EGFR inhibitors. Oncologist. 2019;24: e1417.

2. Magnuson WJ, Lester-Coll NH, Wu AJ, et al. Management of brain metastases in tyrosine kinase inhibitor-naïve epidermal growth factor receptormutant non-small-cell lung cancer: a retrospective multi-institutional analysis. J Clin Oncol. 2017;35:1070-7.

3. Lee CC, Hsu S, Lin CJ, et al. Epidermal growth factor receptor mutations: association with favorable local tumor control following Gamma Knife radiosurgery in patients with non-small cell lung cancer and brain metastases. J Neurosurg. 2019. https://doi.org/10.3171/2019.4.JNS19446.

4. Li C, Fang R, Sun Y, et al. Spectrum of oncogenic driver mutations in lung adenocarcinomas from East Asian never smokers. PLoS ONE. 2011;6: e28204.

5. Eichler AF, Kahle KT, Wang DL, et al. EGFR mutation status and survival after diagnosis of brain metastasis in nonsmall cell lung cancer. Neuro Oncol. 2010;12:1193-9.

6. Jiang T, SU C, Li X, et al. EGFR TKIs plus WBRT demonstrated no survival benefit other than that of TKIs alone in patients with NSCLC and EGFR mutation and brain metastases. J Thorac Oncol. 2016;11:1718-28.

7. Dong K, Liang W, Zhao S, et al. EGFR-TKI plus brain radiotherapy versus EGFR-TKI alone in the management of EGFR-mutated NSCLC patients with brain metastases. Transl Lung Cancer Res. 2019;8:268-79.

8. Gerber NK, Yamada Y, Rimner A, et al. Erlotinib versus radiation therapy for brain metastases in patients with EGFR-mutant lung adenocarcinoma. Int J Radiat Oncol Biol Phys. 2014;89:322-9.

9. Magnuson WJ, Yeung JT, Guillod PD, et al. Impact of deferring radiation therapy in patients with epidermal growth factor receptor-mutant nonsmall cell lung cancer who develop brain metastases. Int J Radiat Oncol Biol Phys. 2016;95:673-9.

10. Ballard P, Yates JW, Yang Z, et al. Preclinical comparison of osimertinib with other EGFR-TKIs in EGFR-mutant NSCLC brain metastases models, and early evidence of clinical brain metastases activity. Clin Cancer Res. 2016;22:5130-40.

11. Cho JH, Lim SH, An HJ, et al. Osimertinib for patients with non-small-cell lung cancer harboring uncommon egfr mutations: a multicenter, openlabel, phase II trial (KCSG-LU15-09). J Clin Oncol. 2020;38:488-95.

12. Brown PD, Ahluwalia MS, Khan $\mathrm{OH}$, et al. Whole-brain radiotherapy for brain metastases: evolution or revolution. J Clin Oncol. 2018;36:483-91.

13. Rades $D$, Pluemer $A$, Veninga $T$, et al. A boost in addition to whole-brain radiotherapy improves patient outcome after resection of 1 or 2 brain metastases in recursive partitioning analysis class 1 and 2 patients. Cancer. 2007;110:1551-9.

14. Du TQ, Li X, Zhong WS, et al. Brain metastases of lung cancer: comparison of survival outcomes among whole brain radiotherapy, whole brain radiotherapy with consecutive boost, and simultaneous integrated boost. J Cancer Res Clin Oncol. 2021;147:569-77.

15. Zimmermann S, Dziadziuszko R, Peters S. Indications and limitations of chemotherapy and targeted agents in non-small cell lung cancer brain metastases. Cancer Treat Rev. 2014:40:716-22.

16. Chen $\mathrm{CH}$, Lee HH, Chuang HY, et al. Combination of whole-brain radiotherapy with epidermal growth factor receptor tyrosine kinase inhibitors improves overall survival in EGFR-mutated non-small cell lung cancer patients with brain metastases. Cancers. 2019;11:1092.

17. Miyawaki E, Kenmotsu H, Mori K, et al. Optimal sequence of local and EGFR-TKI therapy for EGFR-mutant non-small cell lung cancer with brain metastases stratified by number of brain metastases. Int J Radiat Oncol Biol Phys. 2019;104:604-13.

18. Chang EL, Wefel JS, Hess KR, et al. Neurocognition in patients with brain metastases treated with radiosurgery or radiosurgery plus whole-brain irradiation: a randomised controlled trial. Lancet Oncol. 2009;10:1037-44.

19. Khalifa J, Amini A, Popat S, et al. Brain metastases from NSCLC: radiation therapy in the era of targeted therapies. J Thorac Oncol. 2016;11:1627-43. 
20. Ni M, Liu W, Jiang A, et al. Whole brain radiation therapy plus focal radiation boost may generate better survival benefit for brain metastases from non-small cell lung cancer. Front Oncol. 2020;10: 576700.

21. Popp I, Rau S, Hintz M, et al. Hippocampus-avoidance whole-brain radiation therapy with a simultaneous integrated boost for multiple brain metastases. Cancer. 2020;126:2694-703.

22. Lin B, Huang D, Du H, et al. Whole-brain radiation therapy with simultaneous integrated boost versus whole-brain radiation therapy plus stereotactic radiosurgery for the treatment of brain metastasis from lung cancer. Front Oncol. 2021;11:631422.

23. Sperduto PW, Yang TJ, Beal K, et al. Estimating survival in patients with lung cancer and brain metastases: an update of the graded prognostic assessment for lung cancer using molecular markers (Lung-molGPA). JAMA Oncol. 2017;3:827-31.

24. Li H, Lian J, Han S, et al. Applicability of graded prognostic assessment of lung cancer using molecular markers to lung adenocarcinoma patients with brain metastases. Oncotarget. 2017;8:70727-35.

25. Chinnaiyan $\mathrm{P}$, Huang $\mathrm{S}$, Vallabhaneni $\mathrm{G}$, et al. Mechanisms of enhanced radiation response following epidermal growth factor receptor signaling inhibition by erlotinib (Tarceva). Cancer Res. 2005;65:3328-35.

26. Zhao B, Wang Y, Wang Y, et al. Efficacy and safety of therapies for EGFRmutant non-small cell lung cancer with brain metastasis: an evidencebased Bayesian network pooled study of multivariable survival analyses. Aging (Albany NY). 2020;12:14244-70.

27. Chen K, Yu X, Zhang F, et al. Applicability of the Lung-molGPA index in non-small cell lung cancer patients with different gene alterations and brain metastases. Lung Cancer. 2018;125:8-13.

28. Singh R, Lehrer EJ, Ko S, et al. Brain metastases from non-small cell lung cancer with EGFR or ALK mutations: a systematic review and meta-analysis of multidisciplinary approaches. Radiother Oncol. 2020;144:165-79.

29. Ramalingam SS, Vansteenkiste J, Planchard D, et al. Overall survival with osimertinib in untreated, EGFR-mutated advanced NSCLC. N Engl J Med. 2020;382:41-50.

30. Hendriks LE, Smit EF, Vosse BA, et al. EGFR mutated non-small cell lung cancer patients: more prone to development of bone and brain metastases. Lung Cancer. 2014;84:86-91.

31. Aoyama $\mathrm{H}$, Tago M, Shirato $\mathrm{H}$. stereotactic radiosurgery with or without whole-brain radiotherapy for brain metastases: secondary analysis of the JROSG 99-1 randomized clinical trial. JAMA Oncol. 2015;1:457-64.

32. Kraft J, Zindler J, Minniti G, et al. Stereotactic radiosurgery for multiple brain metastases. Curr Treat Options Neurol. 2019;21:6.

33. Yamamoto M, Serizawa T, Shuto T, et al. Stereotactic radiosurgery for patients with multiple brain metastases (JLGK0901): a multi-institutional prospective observational study. Lancet Oncol. 2014;15:387-95.

34. Ballard P, Yates JW, Yang Z, et al. Preclinical comparison of osimertinib with other EGFR-TKIs in EGFR-mutant NSCLC brain metastases models, and early evidence of clinical brain metastases activity. Clin Cancer Res. 2016;22:5130-40.

35. Remon J, Steuer CE, Ramalingam SS, et al. Osimertinib and other third-generation EGFR TKI in EGFR-mutant NSCLC patients. Ann Oncol. 2018;29:i20-7.

36. Yang JJ, Zhou C, Huang Y, et al. Icotinib versus whole-brain irradiation in patients with EGFR-mutant non-small-cell lung cancer and multiple brain metastases (BRAIN): a multicentre, phase 3, open-label, parallel, randomised controlled trial. Lancet Respir Med. 2017:5:707-16.

\section{Publisher's Note}

Springer Nature remains neutral with regard to jurisdictional claims in published maps and institutional affiliations.

Ready to submit your research? Choose BMC and benefit from:

- fast, convenient online submission

- thorough peer review by experienced researchers in your field

- rapid publication on acceptance

- support for research data, including large and complex data types

- gold Open Access which fosters wider collaboration and increased citations

- maximum visibility for your research: over 100M website views per year

At BMC, research is always in progress.

Learn more biomedcentral.com/submissions 